\title{
Predicting Core Level Photoelectron Spectra of Amino Acids Using Density Functional Theory
}

\author{
Jo M. Pi, ${ }^{\dagger}$ Martina Stella, ${ }^{\dagger}$ Nathalie K. Fernando, ${ }^{\ddagger}$ Aaron Y. Lam, ${ }^{\dagger}$ Anna \\ Regoutz, ${ }^{*,+}$ and Laura E. Ratcliff*, \\ $\dagger$ Department of Materials, Imperial College London, London SW7 2AZ, United Kingdom. \\ $\ddagger$ Department of Chemistry, University College London, 20 Gordon Street, London, \\ WC1H 0AJ, United Kingdom.
}

E-mail: a.regoutz@ucl.ac.uk; laura.ratcliff08@imperial.ac.uk

\section{Abstract}

Core level photoelectron spectroscopy is a widely used technique to study amino acids. Interpretation of the individual contributions from functional groups and their local chemical environments to overall spectra requires both high-resolution reference spectra and theoretical insights, for example from density functional theory calculations. This is a particular challenge for crystalline amino acids due to the lack of experimental data and the limitation of previous calculations to gas phase molecules.

Here, a state of the art multiresolution approach is used for high precision gas phase calculations and to validate core hole pseudopotentials for plane-wave calculations. This powerful combination of complementary numerical techniques provides a framework for accurate $\triangle$ SCF calculations for molecules and solids in systematic basis sets. It is used to successfully predict $\mathrm{C}$ and $\mathrm{O} 1 \mathrm{~s}$ core level spectra of glycine, alanine and serine and identify chemical state contributions to experimental spectra of crystalline amino acids.

\section{Graphical TOC Entry}

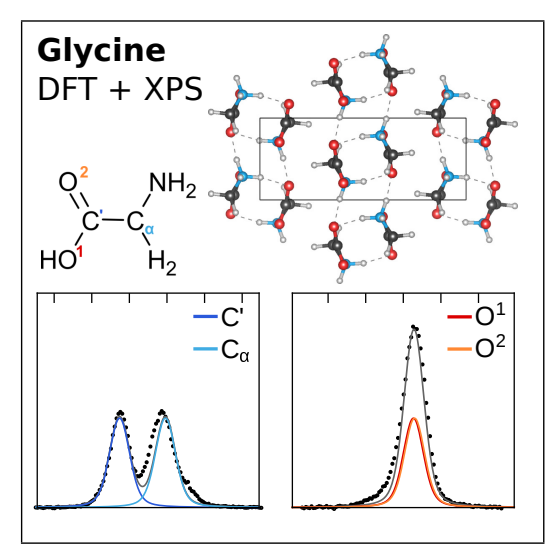


Amino acids have been the subject of investigation for photoelectron spectroscopy (PES) since the very beginning of the technique in the $1970 \mathrm{~s}^{1,2}$. Most experiments focus on gas phase measurements, using He I and II lab sources or soft X-ray synchrotron beamlines as excitation sources $^{3-5}$, or amino acids adsorbed on metallic substrates such as $\mathrm{Cu}, \mathrm{Pd}$ and $\mathrm{Ni}^{6-8}$. In contrast, high-resolution studies on crystalline amino acids in the solid state are scarce ${ }^{9,10}$. One of the primary obstacles for the collection of spectra on solid state amino acids are the effects of radiation damage ${ }^{11,12}$. The advent of scanning abilities in X-ray photoelectron spectrometers coupled with more efficient detectors leading to much faster aquisition times enables a near complete elimination of irradiationinduced defects in spectra. This in turn opens up the possibility to study core states in much more detail and compare results directly to theoretical calculations.

The study of crystalline amino acids, rather than gas or adsorbate phases, is of great importance for a number of reasons. Crystalline amino acids have direct applications as drugs, but also act as important model systems to understand interactions in biomolecules and the folding behaviour of peptides ${ }^{13}$.

Density functional theory ${ }^{14,15}$ is the most widely used electronic structure approach due to its balance between accuracy and efficiency. The simplest approach to calculating core binding energies (BEs) using DFT is the so-called Koopman's approach, wherein the negative value of the Kohn-Sham eigenvalue of the relevant core state is used as a first approximation to the BE. Such an approach neglects final state effects, while it is also only valid for shifts of initial state BEs and not absolute values ${ }^{16,17}$. A more rigorous approach, which also takes into account final state effects, is the $\triangle \mathrm{SCF}$ approach, wherein the BE is defined as

$$
\mathrm{BE}=E_{\text {final }}^{N-1}-E_{\text {initial }}^{N},
$$

where $E_{\text {initial }}^{N}$ is the ground state energy and $E_{\text {final }}^{N-1}$ is the energy of the final state in the presence of a core hole. The $\triangle \mathrm{SCF}$ approach has been used with wide success ${ }^{16,18-20}$, how- ever, the majority of calculations have focused on gas phase molecules, with few examples of applications to periodic systems (see Ref. ${ }^{20}$ and references therein). In the case of amino acids, there are some examples of BE calculations in solution $^{21-23}$, while a cluster approach has been used to model solid state arginine ${ }^{24}$. However, the majority of DFT-based studies focus on gas phase confomers ${ }^{4,25-28}$.

An important challenge for core hole calculations of molecules in widely used Gaussian basis sets is the strong dependence on the choice of basis set ${ }^{28-30}$. Convergence to the basis set limit requires either large basis sets which are prohibitively expensive for large molecules, or the generation of purpose-designed basis sets ${ }^{30}$. This also complicates the comparison of different exchange correlation functionals, due to potential error cancellations between basis set and functional $^{28}$. On the other hand, multiwaveletbased approaches, as implemented in e.g. MADNESS $^{31,32}$ and MRChem $^{33}$, offer systematic convergence. Unlike other systematic basis set approaches such as plane waves, which require the use of pseudopotentials (PSPs), multiwavelets can be used within an all electron (AE) approach, retaining direct access to core electrons. Multiwavelets also permit the definition of a multiresolution approach, wherein the basis is dynamically refined to give high resolution where needed, e.g. close to the nuclei, with lower resolution elsewhere. This balances computational efficiency and precision without requiring specialist user input, and has been demonstrated to give $\mu \mathrm{Ha}$ accuracy ${ }^{34,35}$.

The MADNESS molecular DFT code ${ }^{36-38}$ allows both AE and PSP calculations, as well as a mixed approach in which select atoms are treated at the AE level and the remainder at the PSP level ${ }^{39}$. We have therefore implemented core hole calculations in MADNESS, wherein the core hole is generated by specifying the initial occupancy, following which no additional constraints are imposed. In order to calculate the $\triangle \mathrm{SCF} \mathrm{BE}$ of a given atom in the mixed AE/PSP approach, both a ground state and core hole calculation are performed in which that atom is treated as AE and all others as PSP. This requires multiple ground state calcu- 
lations, however for large molecules the overall computational cost is lower than in the pure AE approach. Furthermore, the mixed approach ensures the core hole remains localized on the atom of interest, avoiding problems with core hole hopping or delocalization ${ }^{28,40}$. Taking the example of methanol, the BEs calculated using the mixed approach are within a few tenths of an $\mathrm{eV}$ of the pure AE values, while the accuracy is comparable to that of a large Gaussian basis set (see Supplementary Information).

In this work we explore in depth the calculation of $\mathrm{C}$ and $\mathrm{O} 1 \mathrm{~s}$ BEs and compare them to core level X-ray photoelectron spectroscopy of the amino acids glycine (Gly), alanine (Ala) and serine (Ser), depicted in Fig. 1. Spectra were recorded on a Thermo Scientific K-Alpha+ X-ray Photoelectron Spectrometer (XPS) using a monochromated, microfocused $\mathrm{Al} \mathrm{K} \alpha$ X-ray source $(\mathrm{h} \nu=1486.7 \mathrm{eV})$. Further measurement and computational details can be found in the Supplementary Information (SI).

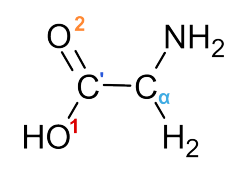

(a) Gly

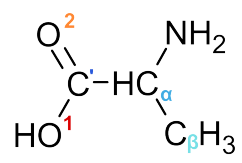

(b) Ala

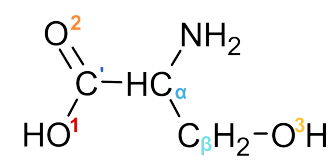

(c) Ser
Figure 1: Schematic of Gly, Ala and Ser, showing the atomic structures and atom labels which will be used in the following.

In the first instance we consider gas phase amino acids. A range of low energy confomers for Gly, Ala and Ser were taken from the literature $^{28,41}$. The confomers were relaxed with BigDFT $^{42}$ using $\mathrm{PBE}^{43}$, as described in the SI, and the relaxed atomic structures are depicted in Fig. 2. For each conformer the BEs were calculated using both the Koopman's and $\triangle \mathrm{SCF}$ approaches for a range of exchange-correlation functionals: the local density approximation $(\mathrm{LDA})^{44,45}$, two generalized gradient approximations, namely $\mathrm{PBE}$ and $\mathrm{BLYP}^{46,47}$, and two hybrid functionals, namely $\mathrm{PBE}^{48}$ and B3LYP $^{45,47,49,50}$.

In order to evaluate the applicability of the various functionals, Gly and Ala were explored since gas phase experimental XPS data is available for comparison ${ }^{3,4}$. Calculated $\mathrm{C}$ and $\mathrm{O} 1 \mathrm{~s}$

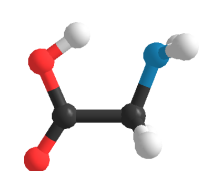

(a) Gly 1

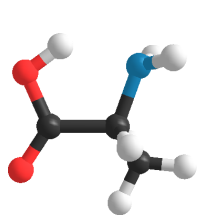

(e) Ala 1

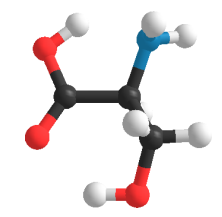

(i) Ser 1

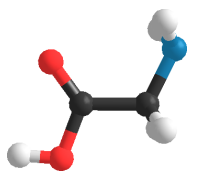

(b) Gly 2

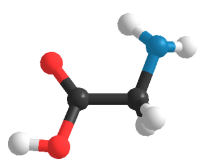

(c) Gly 3

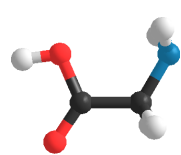

(d) Gly 4

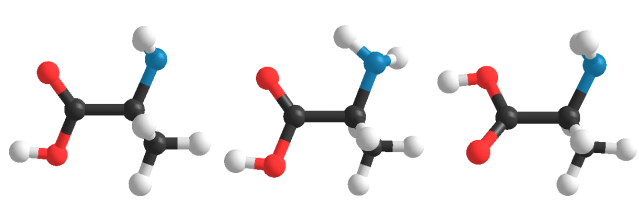

(f) Ala 2

(g) Ala 3

(h) Ala 4

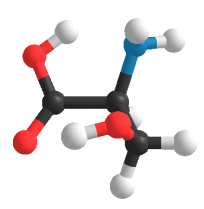

(j) Ser 2

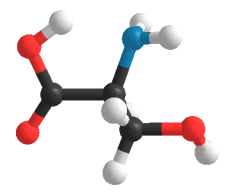

(k) Ser 3

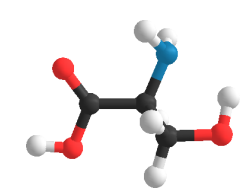

(1) Ser 4

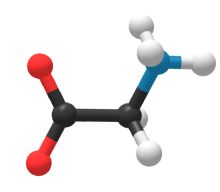

(n) Gly z

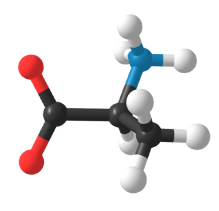

(o) Ala z

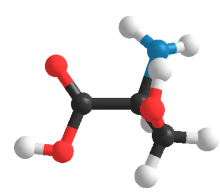

(m) Ser 5

Figure 2: Relaxed atomic structures of the studied confomers of the gas phase amino acids, and the zwitterionic (' $z$ ') structures as extracted from the relaxed crystals. $\mathrm{C} / \mathrm{O} / \mathrm{N} / \mathrm{H}$ atoms are depicted in grey/red/blue/white.

core level BEs are sensitive to the level of theory, but while $\mathrm{O} 1 s$ shows no significant differences between functionals, $\mathrm{C} 1 s$ displays considerable changes. Relative BEs for Gly, Ala and Ser and comparative plots of the theoretical results and available literature gas phase data are included in the SI. For all cases $\triangle \mathrm{SCF}$ relative BEs are more consistent with experimental results. The best description of the experimental data available is provided by using either B3LYP or PBE0 functionals with the $\triangle \mathrm{SCF}$ approach. This agrees with previous work where PBE0 and $\mathrm{PBEOP}^{51}$ gave the best performance for several amino acids when using large basis 
$\operatorname{sets}^{28}$. Based on these results, only PBE0 with $\triangle \mathrm{SCF}$ was used to explore differences between gas phase confomers in detail.

Whilst highly accurate gas phase calculations are accessible at low computational cost using the multiwavelet approach, there is a strong motivation to predict experimental results for solid crystalline samples. In the crystalline form amino acids are present in their zwitterionic state, which is not the case in the gas phase. This is expected to have a big impact in particular on the $\mathrm{O} 1 s$ BEs. To test the applicability of the different structural models, results from gas phase, isolated zwitterion and periodic solid calculations are compared to experimental data.

Theoretical gas phase results for the different confomers were compared to the experimental results for solid amino acids (see Fig. 3). Gly and Ala give similar results where confomers 1 of both, in which the hydrogen of the carboxylic group points towards the amino group, give $\mathrm{C} 1 s \mathrm{BEs}$ close to the experimental solid values. Confomers 2-4 are distinctly different from confomer 1 , but give relative BEs within $0.1 \mathrm{eV}$ of each other, which agree with the experimental gas phase rather than solid results. A similar trend holds for Ser, however, the overall discrepancy between gas phase theory and experiment is greater. As expected from the difference in atomic structure the calculated $\mathrm{O} 1 \mathrm{~s}$ BEs for all gas phase confomers vary distinctly from the experimental results. In addition, Ser O $1 s$ BEs show greater variations between the confomers.

In contrast to the confomers considered so far, zwitterion structures are not stable in the gas phase at the level of DFT. Instead, unrelaxed zwitterions can be calculated by first optimizing the geometry of the crystal structure, then extracting a single molecule as shown in Figs. 2(n), 2(o) and 2(p). Initial crystal structures were taken from Refs. ${ }^{52-54}$ for Gly, Ala and Ser respectively, and the unit cells were relaxed using CASTEP ${ }^{55}$, as described in the SI. The final lattice parameters were found to be within $4 \%$ of the experimental values and the relaxed structures are depicted in Fig. 4.

Upon comparison of the zwitterion results

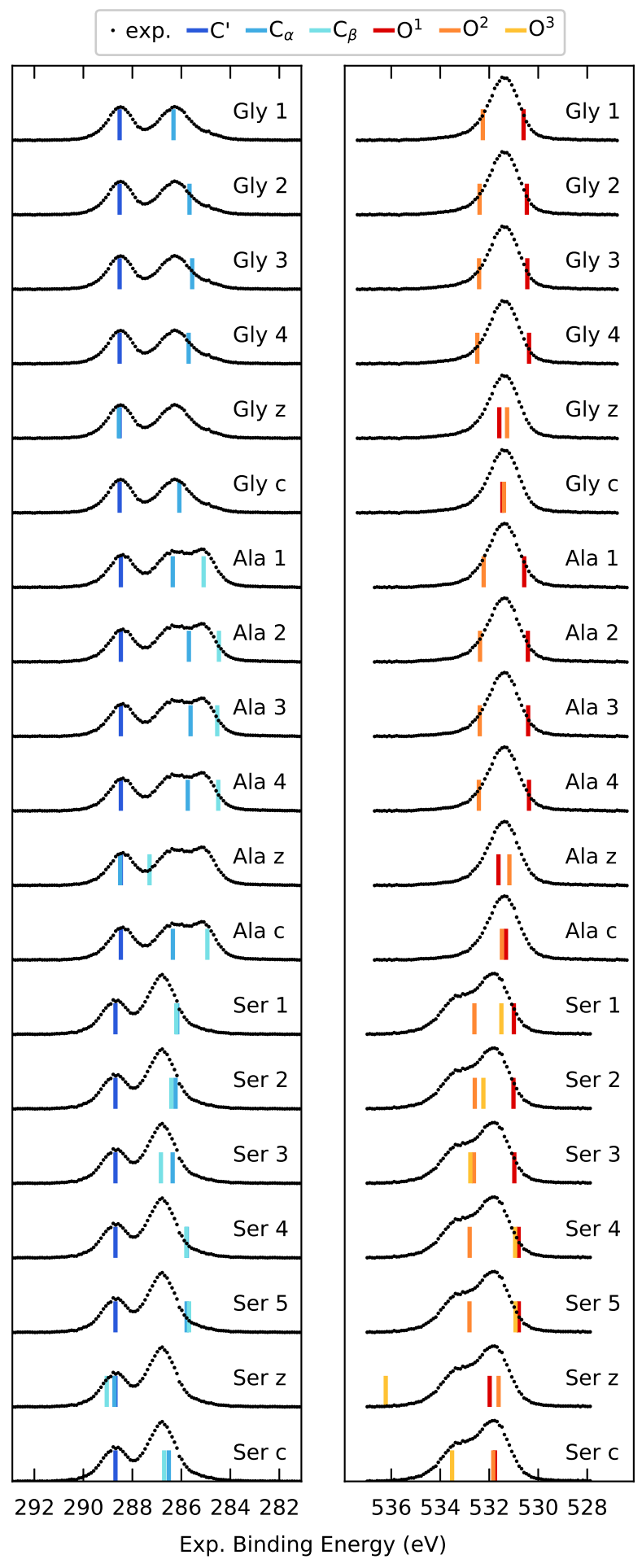

Figure 3: Comparison of solid state experimental $\mathrm{C}$ and $\mathrm{O} 1 s$ core level spectra (points) and theoretical BEs (lines) for gas phase confomers, zwitterions ('z') and crystals ('c') calculated using the $\triangle \mathrm{SCF}$ approach with PBE0. The calculated $\mathrm{C}^{\prime} \mathrm{BE}$ was used to align to experiment, while the $\mathrm{O} 1 s$ spectra were aligned by centering the $\mathrm{O}^{1}$ and $\mathrm{O}^{2}$ peaks around the experimental peak. 


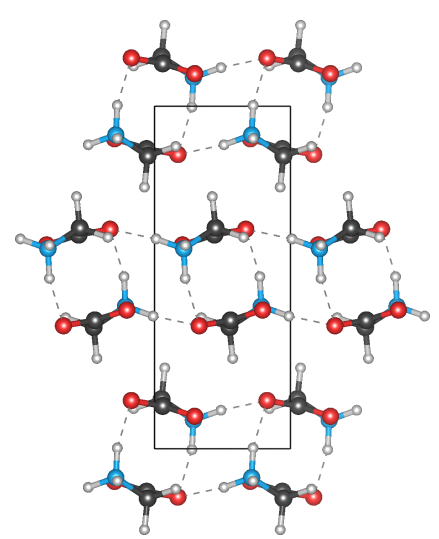

(a) Gly

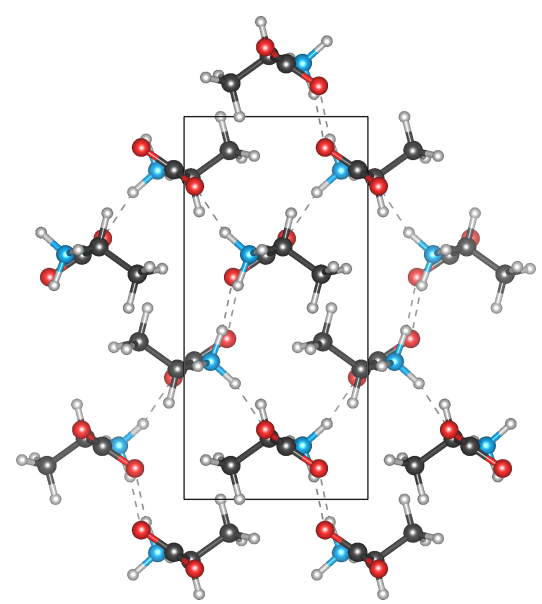

(b) Ala

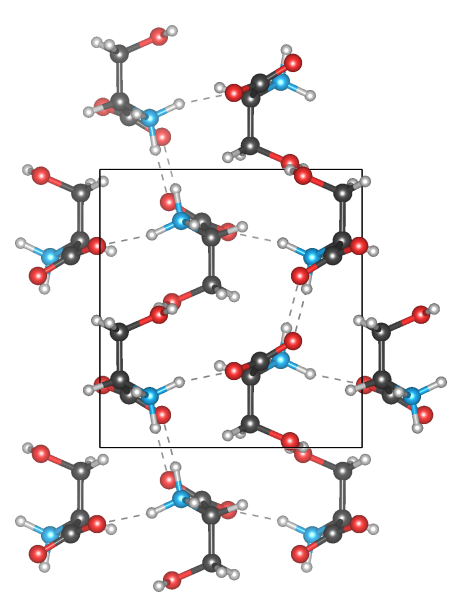

(c) Ser

Figure 4: Crystal structures of the three amino acids as viewed along the $c$ axis, where the black lines indicate the unit cell boundaries. $\mathrm{C} / \mathrm{O} / \mathrm{N} / \mathrm{H}$ atoms are depicted in grey/red/blue/white. Images viewed along the other axes are shown in the SI.

with the experiment it is obvious that the $\mathrm{C} 1 \mathrm{~s}$ BEs diverge dramatically from the experimentally observed values. This discrepancy can be explained by the lack of intermolecular interactions, which become important in the presence of charged functional groups. However, the description of the $\mathrm{O} 1 s \mathrm{BEs}$ of the carboxylic group is improved compared to the gas phase results, as the zwitterion provides a closer approximation of the solid state.

Whilst gas phase and zwitterion models can provide some insight for the interpretation of experimental results, it is clear that calculations beyond the gas phase are necessary to accurately calculate BEs of solid amino acids. A common approach to calculating BEs of periodic structures is to generate clusters ${ }^{20,56,57}$. This strategy was tested by extracting molecular clusters from the bulk structures and calculating BEs of the central molecule. However, the BEs converge slowly with cluster size, e.g. for Gly an $8 \AA$ radius is insufficient, which is consistent with QM/MM calculations for Gly in water ${ }^{22}$. Furthermore, core hole calculations for molecular clusters in MADNESS converged more slowly and unpredictably compared to the gas phase. Based on these limitations BEs were calculated directly in a periodic crystal.

Plane-waves provide a natural systematic basis set for calculations in periodic crystals.
While the core electrons are only treated implicitly through the use of PSPs, it is straightforward to generate a PSP which incorporates a core hole within CASTEP $^{58}$. The key factor influencing the accuracy of such an approach is the PSP. These were validated using MADNESS as described in the SI. As with the gas phase calculations, only relative BEs were considered, following which the theoretical spectra were manually aligned with experiment. This avoids for example the need to account for the chemical potential (as described e.g. in Ref. ${ }^{59}$ ) and reduces the computational cost, since the relative BEs converge more quickly with respect to supercell size than absolute BEs.

CASTEP with PBE0 gives core level BEs for both $\mathrm{C}$ and $\mathrm{O} 1 s$ which are in good agreement with experimental results (see Fig. 3). The significant improvement compared to the other presented approaches shows that it is necessary to combine both the correct zwitterionic structure of the molecule with the incorporation of intermolecular interactions.

Based on the BE values from theory spectral line shapes are calculated (see Fig. 5(a)). A Voigt function consisting of $0.44 \mathrm{eV}$ FWHM Gaussian and 0.20 eV FWHM Lorentzian contributions was applied. The $0.44 \mathrm{eV}$ Gaussian contribution is consistent with the experimental resolution determined from a reference mea- 
surement of the Fermi edge of a gold foil. The calculated spectral lineshapes describe the BE positions of the individual contributions as well as their relative intensities of the observed experimental spectra very well. In order to compare the experimentally observed BEs with the theoretically predicted values, peak fit analysis of the core levels was performed (see Fig. 5(b) and details in SI). Two features observed in the experimental spectra are not included in the theoretical calculations. In the $\mathrm{C} 1 s$ spectra a small contribution from adventitious carbon is observed at a $\mathrm{BE}$ of $285.2 \mathrm{eV}$, which in Ala overlaps with the contribution from the aliphatic $\mathrm{C}_{\beta}$. In the $\mathrm{O} 1 \mathrm{~s}$ spectra features at higher BEs compared to the main carboxylic and hydroxyl contributions stem from surface species, including adsorbed water. The theoretically predicted core level spectra enable the identification of specific chemical states within the amino acids, forgoing the need for interpretation of spectra solely based on literature references and basic chemical arguments. Furthermore, they can help identify non-intrinsic spectral contributions, including surface species as mentioned above.

Tab.1 summarises the experimental BEs extracted from peak fit analysis and theoretical BEs from solid state $\triangle \mathrm{SCF}$ calculations using PBE and PBE0. Based on the gas phase calculations PBE0 was found to give the best results, however, the increase in computational cost compared to PBE is significant, particularly for solid state calculations. Therefore, it is useful to investigate whether this increased cost is justified by an increase in accuracy, so BEs were calculated with both PBE and PBE0. No clear trend can be observed to suggest that either of the two functionals provides a better description of the experimental observations. For $\mathrm{C} 1 s$ PBE underestimates whilst PBE0 overestimates the magnitude of the relative BEs. As with the gas phase there is little difference between the theoretical results for $\mathrm{O} 1 s$.

In conclusion, this work shows that the $\triangle \mathrm{SCF}$ approach as implemented within a systematic basis set provides a direct way to predict the core levels and all contributing chemical states of amino acids. The calculated spectra can be
Table 1: Comparison of experimental BEs and theoretical BEs from solid state $\triangle$ SCF calculations using PBE and PBE0. Relative BEs are with respect to $\mathrm{C}^{\prime}$ or $\mathrm{O}^{1}$ of the given system. For experimental core levels n.d. (not detected) is used to indicate overlapping contributions. All energies are in $\mathrm{eV}$.

\begin{tabular}{|c|c|c|c|c|c|}
\hline & & \multirow{3}{*}{$\begin{array}{c}\mathrm{BE} \\
\text { Exp. }\end{array}$} & \multicolumn{3}{|c|}{ Relative BE } \\
\hline & & & \multirow[t]{2}{*}{ Exp. } & \multicolumn{2}{|c|}{ Theor. } \\
\hline & & & & PBE & PBE0 \\
\hline \multirow[t]{4}{*}{ Gly } & $\mathrm{C}^{\prime}$ & 288.5 & - & - & - \\
\hline & $\mathrm{C}^{\alpha}$ & 286.3 & -2.2 & -2.02 & -2.44 \\
\hline & $\mathrm{O}^{1}$ & 531.4 & - & $\begin{array}{lll}- & \text { r }\end{array}$ & - \\
\hline & $\mathrm{O}^{2}$ & 531.4 & n.d. & -0.06 & -0.06 \\
\hline \multirow[t]{5}{*}{ Ala } & $\mathrm{C}^{\prime}$ & 288.5 & - & - & - \\
\hline & $\mathrm{C}^{\alpha}$ & 286.5 & -2.0 & -1.78 & -2.12 \\
\hline & $\mathrm{C}^{\beta}$ & 285.2 & -3.3 & -3.09 & -3.53 \\
\hline & $\mathrm{O}^{1}$ & 531.4 & - & - & - \\
\hline & $\mathrm{O}^{2}$ & 531.4 & n.d. & 0.17 & 0.17 \\
\hline \multirow[t]{6}{*}{ Ser } & $\mathrm{C}^{\prime}$ & 288.8 & - & - & - \\
\hline & $\mathrm{C}^{\alpha}$ & 286.9 & -1.9 & -1.86 & -2.18 \\
\hline & $\mathrm{C}^{\beta}$ & 286.9 & -1.9 & -1.65 & -1.99 \\
\hline & $\mathrm{O}^{1}$ & 531.8 & - & - & - \\
\hline & $\mathrm{O}^{2}$ & 531.8 & n.d. & 0.08 & 0.07 \\
\hline & $\mathrm{O}^{3}$ & 533.2 & 1.4 & 1.64 & 1.68 \\
\hline
\end{tabular}

directly compared to measured X-ray photoelectron spectra of solid samples, which enables the identification of individual contributions to the experimentally observed line shapes. The enhanced understanding of XP spectra achieved by comparison to results from the novel computational approach taken in this work provides new insights into the exact chemical nature of amino acids in the crystalline state. This places XPS in a position to be a powerful characterisation technique for crystalline amino acids, complementing structural techniques. The successful combination of theory and experiment employed here to understand amino acids can be further extended to other small molecular systems, where a detailed exploration of core states can provide invaluable information to understand their chemistry.

Acknowledgement LER and MS acknowledge support from an EPSRC Early Career 


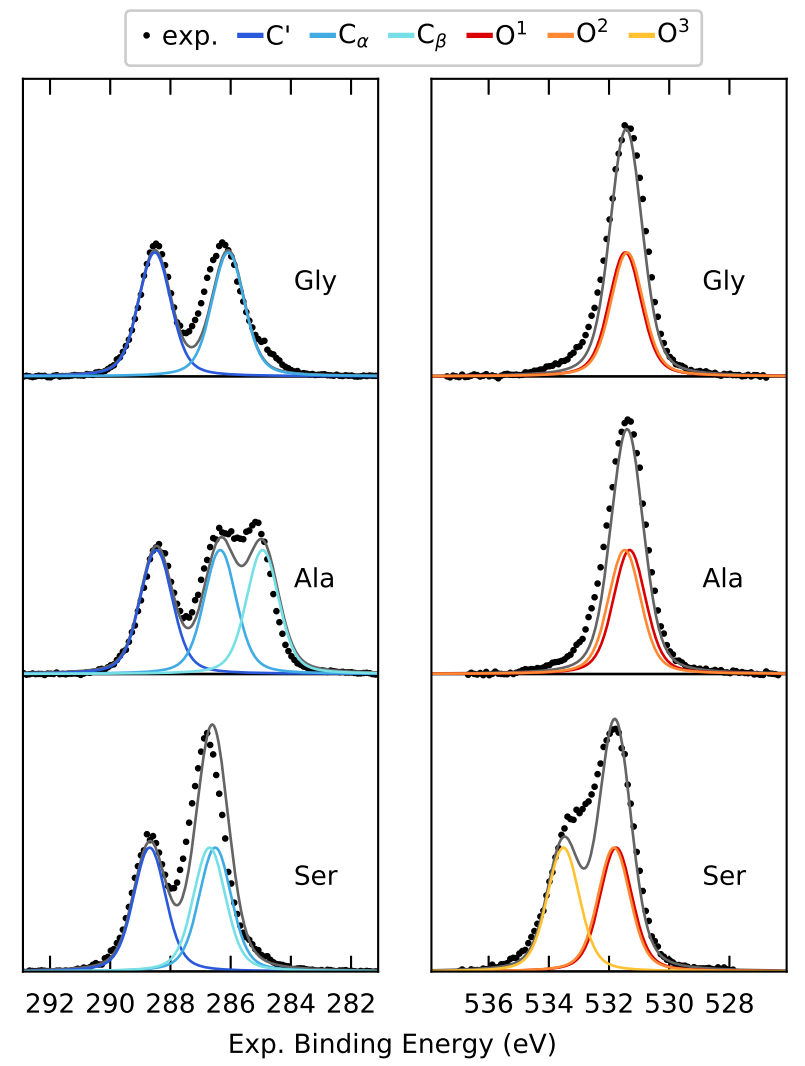

(a) Theoretical results including Voigt broadening (lines) and experimental spectra (points).

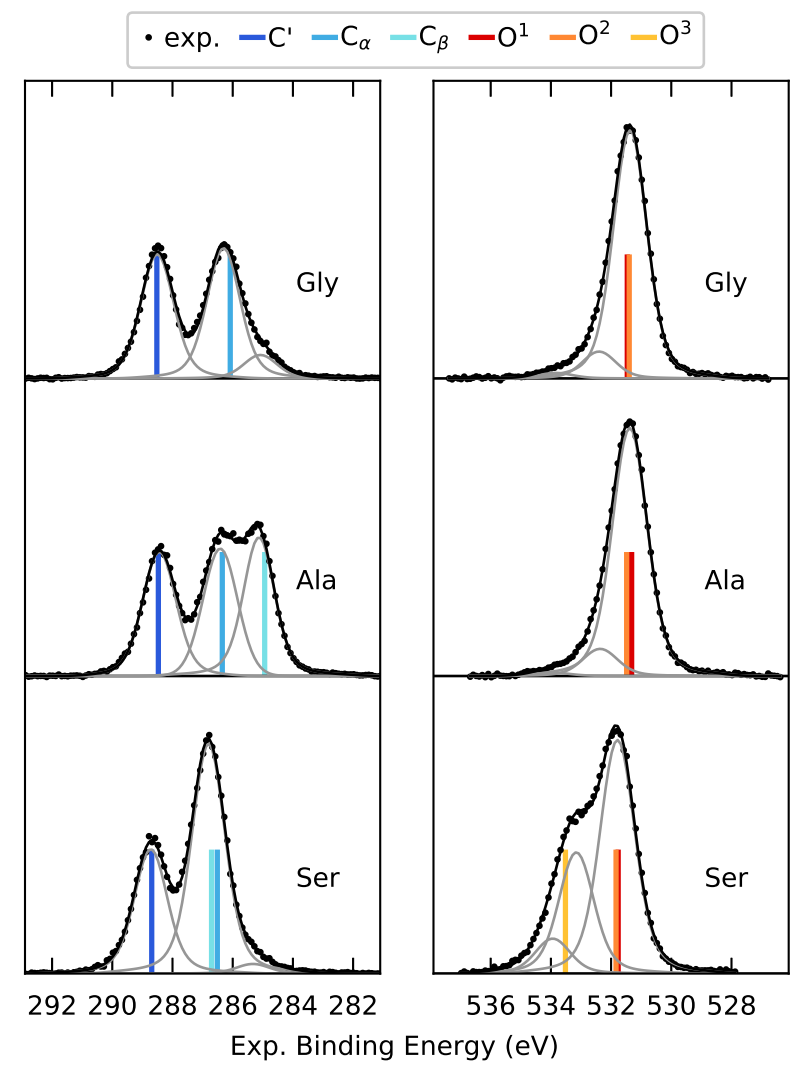

(b) Peak fits (lines) of experimental data (points). Theoretical values are depicted as coloured vertical lines.

Figure 5: Comparison of experimental and theoretical spectra, where calculations have been performed using the $\triangle \mathrm{SCF}$ approach in a periodic crystal using the PBE0 functional. The theoretical results have been aligned as described in Fig. 3.

Research Fellowship (EP/P033253/1) and the Thomas Young Centre under grant number TYC-101. AR acknowledges support from the Analytical Chemistry Trust Fund for her CAMS-UK Fellowship and from Imperial College London for her Imperial College Research Fellowship. AR and AYL acknowledge funding from the Analytical Chemistry Trust Fund for an Analytical Chemistry Summer Studentship. NF acknowledges support from the Engineering and Physical Sciences Research Council (EP/L015277/1). Calculations were performed on the Imperial College High Performance Computing Service and the ARCHER UK National Supercomputing Service. LER thanks Fabiano Corsetti for useful discussions. Computational and experimental details, additional results and the relaxed atomic structures of the molecules and crystals are available in the Sup- plementary Information. The Supplementary Information contains additional references, particularly pertaining to the computational methods used ${ }^{60-70}$.

\section{References}

(1) Clark, D.; Peeling, J.; Colling, L. An experimental and theoretical investigation of the core level spectra of a series of amino acids, dipeptides and polypeptides. Biochim. Biophys. Acta 1976, 453, 533.

(2) Cannington, P. H.; Ham, N. S. The photoelectron spectra of amino-acids: A survey. J. Electron Spectrosc. 1979, 15, 79.

(3) Slaughter, A. R.; Banna, M. S. Corephotoelectron binding energies of gaseous 
glycine: correlation with its proton affinity and gas-phase acidity. J. Phys. Chem. 1988, 92, 2165-2167.

(4) Feyer, V.; Plekan, O.; Richter, R.; Coreno, M.; Prince, K. C.; Carravetta, V. Core level study of alanine and threonine. J. Phys. Chem. A 2008, 112, 7806-7815.

(5) Plekan, O.; Feyer, V.; Richter, R.; Coreno, M.; de Simone, M.; Prince, K. C.; Carravetta, V. Investigation of the amino acids glycine, proline, and methionine by photoemission spectroscopy. J. Phys. Chem. A 2007, 111, 10998.

(6) Eralp, T.; Shavorskiy, A.; Zheleva, Z. V.; Held, G.; Kalashnyk, N.; Ning, Y.; Linderoth, T. R. Global and local expression of chirality in serine on the $\mathrm{Cu}\{110\}$ surface. Langmuir 2010, 26, 18841.

(7) Gao, F.; Li, Z.; Wang, Y.; Burkholder, L.; Tysoe, W. T. Chemistry of alanine on $\operatorname{Pd}(111)$ : temperature-programmed desorption and X-ray photoelectron spectroscopic study. Surf. Sci. 2007, 601, 3276.

(8) Nicklin, R. E. J.; Cornish, A.; Shavorskiy, A.; Baldanza, S.; Schulte, K.; Liu, Z.; Bennett, R. A.; Held, G. Surface chemistry of alanine on $\mathrm{Ni}\{111\}$. J. Phys. Chem. C 2015, 119, 26566.

(9) Stevens, J. S.; de Luca, A. C.; Pelenditris, M.; Terenghi, G.; Downes, S.; Schroeder, S. L. M. Quantitative analysis of complex amino acids and RGD peptides by X-ray photoelectron spectroscopy (XPS). Surf. Interface Anal. 2013, 45, 1238.

(10) Brizzolara, R. A. Methionine by Xray photoelectron spectroscopy. Surf. Sci. Spectra 1996, 4, 96.

(11) Tzvetkov, G.; Netzer, F. P. X-ray induced irradiation effects in glycine thin films: A time-dependent XPS and TPD study. J. Electron. Spectrosc. 2010, 182, 41-46.
(12) Zubavichus, Y.; Zharnikov, M.; Shaporenko, A.; Fuchs, O.; Weinhardt, L.; Heske, C.; Umbach, E.; Denlinger, J. D.; Grunze, M. Soft X-ray Induced Decomposition of Phenylalanine and Tyrosine: A Comparative Study. J. Phys. Chem. A 2004, 108, 4557-4565.

(13) Boldyreva, E. Crystalline amino acids. Models, mysteries and magic of molecules. Dordrecht, 2008; pp 167-192.

(14) Hohenberg, P.; Kohn, W. Inhomogeneous electron gas. Phys. Rev. 1964, 136, B864B871.

(15) Kohn, W.; Sham, L. J. Self-consistent equations including exchange and correlation effects. Phys. Rev. 1965, 140, A1133A1138.

(16) Pueyo Bellafont, N.; Bagus, P. S.; Illas, F. Prediction of core level binding energies in density functional theory: rigorous definition of initial and final state contributions and implications on the physical meaning of Kohn-Sham energies. J. Chem. Phys. 2015, 142, 214102.

(17) Pueyo Bellafont, N.; Illas, F.; Bagus, P. S. Validation of Koopmans' theorem for density functional theory binding energies. Phys. Chem. Chem. Phys. 2015, 17, 4015-4019.

(18) Cavigliasso, G.; Chong, D. P. Accurate density-functional calculation of coreelectron binding energies by a total-energy difference approach. J. Chem. Phys. 1999, 111, 9485-9492.

(19) Besley, N. A.; Gilbert, A. T. B.; Gill, P. M. W. Self-consistent-field calculations of core excited states. J. Chem. Phys. 2009, 130, 124308 .

(20) Viñes, F.; Sousa, C.; Illas, F. On the prediction of core level binding energies in molecules, surfaces and solids. Phys. Chem. Chem. Phys. 2018, 20, 8403-8410. 
(21) Chatterjee, A.; Zhao, L.; Zhang, L.; Pradhan, D.; Zhou, X.; Leung, K. T. Corelevel electronic structure of solid-phase glycine, glycyl-glycine, diglycyl-glycine, and polyglycine: X-ray photoemission analysis and Hartree-Fock calculations of their zwitterions. J. Chem. Phys. 2008, 129, 105104.

(22) Niskanen, J.; Arul Murugan, N.; Rinkevicius, Z.; Vahtras, O.; Li, C.; Monti, S.; Carravetta, V.; Ågren, H. Hybrid density functional-molecular mechanics calculations for core-electron binding energies of glycine in water solution. Phys. Chem. Chem. Phys. 2013, 15, 244-254.

(23) Carravetta, V.; de Abreu Gomes, A. H.; Monti, S.; Mocellin, A.; Marinho, R. R. T.; Björneholm, O.; Ågren, H.; Naves de Brito, A. pH-dependent X-ray photoelectron chemical shifts and suface distribution of cysteine in aqueous solution. J. Phys. Chem. B 2019, 123, 37763785 .

(24) Li, H.; Hua, W.; Lin, Z.; Luo, Y. Firstprinciples study on core-level spectroscopy of arginine in gas and solid phases. $J$. Phys. Chem. B 2012, 116, 12641-12650.

(25) Chong, D. P. Density-functional calculation of core-electron binding energies of glycine conformers. Can. J. Chem. 1996, 74, 1005-1007.

(26) Plekan, O.; Feyer, V.; Richter, R.; Coreno, M.; de Simone, M.; Prince, K.; Carravetta, V. Photoemission and the shape of amino acids. Chem. Phys. Lett. 2007, 442, 429-433.

(27) Tolbatov, I.; Chipman, D. M. In Isaiah Shavitt: A Memorial Festschrift from Theoretical Chemistry Accounts; Shepard, R., Pitzer, R. M., Dunning, T., Eds.; Springer Berlin Heidelberg: Berlin, Heidelberg, 2016; pp 187-193.

(28) Tolbatov, I.; Chipman, D. M. Benchmarking density functionals and Gaussian basis sets for calculation of core-electron binding energies in amino acids. Theor. Chem. Acc. 2017, 136, 82.

(29) Fouda, A. E. A.; Besley, N. A. Assessment of basis sets for density functional theory-based calculations of core-electron spectroscopies. Theor. Chem. Acc. 2017, $137,6$.

(30) Hanson-Heine, M. W.; George, M. W.; Besley, N. A. Basis sets for the calculation of core-electron binding energies. Chem. Phys. Lett. 2018, 699, 279 - 285.

(31) Harrison, R. J.; Beylkin, G.; Bischoff, F. A.; Calvin, J. A.; Fann, G. I.; Fosso-Tande, J.; Galindo, D.; Hammond, J. R.; Hartman-Baker, R.; Hill, J. C. et al. MADNESS: A Multiresolution, Adaptive Numerical Environment for Scientific Simulation. SIAM J. Sci. Comput. 2016, 38, S123-S142.

(32) MADNESS code repository, https://github.com/m-a-d-n-e-s-s/madness.

(33) MultiResolution Chemistry (MRChem) program package, https://mrchem.readthedocs.io/en/latest/.

(34) Jensen, S. R.; Saha, S.; Flores-Livas, J. A.; Huhn, W.; Blum, V.; Goedecker, S.; Frediani, L. The elephant in the room of density functional theory calculations. $J$. Phys. Chem. Lett. 2017, 8, 1449-1457.

(35) Gulans, A.; Kozhevnikov, A.; Draxl, C. Microhartree precision in density functional theory calculations. Phys. Rev. B 2018, 97, 161105.

(36) Harrison, R. J.; Fann, G. I.; Yanai, T.; Beylkin, G. In Computational Science ICCS 2003: International Conference, Melbourne, Australia and St. Petersburg, Russia, June 2-4, 2003 Proceedings, Part $I V$; Sloot, P. M. A., Abramson, D., Bogdanov, A. V., Gorbachev, Y. E., Dongarra, J. J., Zomaya, A. Y., Eds.; Springer Berlin Heidelberg: Berlin, Heidelberg, 2003; pp 103-110. 
(37) Harrison, R. J.; Fann, G. I.; Yanai, T.; Gan, Z.; Beylkin, G. Multiresolution quantum chemistry: basic theory and initial applications. J. Chem. Phys. 2004, 121, 11587-11598.

(38) Yanai, T.; Fann, G. I.; Gan, Z.; Harrison, R. J.; Beylkin, G. Multiresolution quantum chemistry in multiwavelet bases: analytic derivatives for Hartree-Fock and density functional theory. J. Chem. Phys. 2004, 121, 2866-2876.

(39) Ratcliff, L. E.; Thornton, W. S.; Vázquez Mayagoitia, A.; Romero, N. A. Combining pseudopotential and all electron density functional theory for the efficient calculation of core spectra using a multiresolution approach. J. Phys. Chem. A 2019, 123, 4465-4474.

(40) Bagus, P. S.; Schaefer, H. F. Localized and delocalized 1s jole states of the $\mathrm{O} 2+$ molecular ion. J. Chem. Phys. 1972, 56, 224-226.

(41) Miao, R.; Jin, C.; Yang, G.; Hong, J.; Zhao, C.; Zhu, L. Comprehensive density functional theory study on serine and related ions in gas phase: conformations, gas phase basicities, and acidities. J. Phys. Chem. A 2005, 109, 2340-2349.

(42) Genovese, L.; Neelov, A.; Goedecker, S.; Deutsch, T.; Ghasemi, S. A.; Willand, A.; Caliste, D.; Zilberberg, O.; Rayson, M.; Bergman, A. et al. Daubechies wavelets as a basis set for density functional pseudopotential calculations. J. Chem. Phys. 2008, 129, 014109.

(43) Perdew, J. P.; Burke, K.; Ernzerhof, M. Generalized gradient approximation made simple. Phys. Rev. Lett. 1996, 77, 38653868 .

(44) Ceperley, D. M.; Alder, B. J. Ground State of the electron gas by a stochastic method. Phys. Rev. Lett. 1980, 45, 566569 .
(45) Vosko, S. H.; Wilk, L.; Nusair, M. Accurate spin-dependent electron liquid correlation energies for local spin density calculations: a critical analysis. Can. J. Phys. 1980, 58, 1200-1211.

(46) Becke, A. D. Density-functional exchangeenergy approximation with correct asymptotic behavior. Phys. Rev. A 1988, 38, 3098-3100.

(47) Lee, C.; Yang, W.; Parr, R. G. Development of the Colle-Salvetti correlationenergy formula into a functional of the electron density. Phys. Rev. B 1988, 37, 785-789.

(48) Adamo, C.; Barone, V. Toward reliable density functional methods without adjustable parameters: The PBE0 model. $J$. Chem. Phys. 1999, 110, 6158-6170.

(49) Becke, A. D. Density-functional thermochemistry. III. The role of exact exchange. J. Chem. Phys. 1993, 98, 5648-5652.

(50) Stephens, P. J.; Devlin, F. J.; Chabalowski, C. F.; Frisch, M. J. Ab initio calculation of vibrational absorption and circular dichroism spectra using density functional force fields. J. Phys. Chem. 1994, 98, 11623-11627.

(51) Tsuneda, T.; Suzumura, T.; Hirao, K. A new one-parameter progressive Colle-Salvetti-type correlation functional. J. Chem. Phys. 1999, 110, 10664-10678.

(52) Marsh, R. E. A refinement of the crystal structure of glycine. Acta Cryst. 1958, 11, 654-663.

(53) Destro, R.; Marsh, R. E.; Bianchi, R. A low-temperature $(23 \mathrm{~K})$ study of Lalanine. J. Phys. Chem 1988, 92, 966973.

(54) Benedetti, E.; Pedone, C.; Sirigu, A. The crystal structure of L-serine. Gazzetta Chimica Italiana 1973, 103, 555. 
(55) Clark, S. J.; Segall, M. D.; Pickard, C. J.; Hasnip, P. J.; Probert, M. J.; Refson, K.; Payne, M. First principles methods using CASTEP. Z. Kristall. 2005, 220, 567570 .

(56) The interpretation of XPS spectra: Insights into materials properties. Surface Science Reports 2013, 68, 273 - 304.

(57) Kahk, J. M.; Lischner, J. Accurate absolute core-electron binding energies of molecules, solids, and surfaces from firstprinciples calculations. Phys. Rev. Materials 2019, 3, 100801.

(58) Gao, S.-P.; Pickard, C. J.; Perlov, A.; Milman, V. Core-Level spectroscopy calculation and the plane wave pseudopotential method. J. Phys.: Condens. Matter 2009, 21, 104203.

(59) Ozaki, T.; Lee, C.-C. Absolute binding energies of core levels in solids from first principles. Phys. Rev. Lett. 2017, 118, 026401.

(60) Goedecker, S.; Teter, M.; Hutter, J. Separable dual-space Gaussian pseudopotentials. Phys. Rev. B 1996, 54, 1703-1710.

(61) Gronert, S.; O’Hair, R. A. J. Ab initio studies of amino acid conformations. 1. The conformers of alanine, serine, and cysteine. J. Am. Chem. Soc. 1995, 117, 2071-2081.

(62) Pecul, M. Conformational structures and optical rotation of serine and cysteine. Chem. Phys. Lett. 2006, 418, $1-10$.

(63) Yu, W.; Liang, L.; Lin, Z.; Ling, S.; Haranczyk, M.; Gutowski, M. Comparison of some representative density functional theory and wave function theory methods for the studies of amino acids. J. Comp. Chem. 2009, 30, 589-600.

(64) Hartwigsen, C.; Goedecker, S.; Hutter, J. Relativistic separable dual-space Gaussian pseudopotentials from $\mathrm{H}$ to Rn. Phys. Rev. B 1998, 58, 3641-3662.
(65) Anderson, J.; Harrison, R. J.; Sekino, H.; Sundahl, B.; Beylkin, G.; Fann, G. I.; Jensen, S. R.; Sagert, I. On derivatives of smooth functions represented in multiwavelet bases. J. Computat. Physics: $X$ 2019, 4, 100033.

(66) Grimme, S. Semiempirical GGA-type density functional constructed with a longrange dispersion correction. J. Comput. Chem. 2006, 27, 1787.

(67) Monkhorst, H. J.; Pack, J. D. Special points for Brillouin-zone integrations. Phys. Rev. B 1976, 13, 5188-5192.

(68) Valiev, M.; Bylaska, E.; Govind, N.; Kowalski, K.; Straatsma, T.; Dam, H. V.; Wang, D.; Nieplocha, J.; Apra, E.; Windus, T. et al. NWChem: a comprehensive and scalable open-source solution for large scale molecular simulations. Comput. Phys. Commun. 2010, 181, 1477 1489 .

(69) Woon, D. E.; Dunning, T. H. Gaussian basis sets for use in correlated molecular calculations. V. Core-valence basis sets for boron through neon. J. Chem. Phys. 1995, 103, 4572-4585.

(70) Dunning, T. H. Gaussian basis sets for use in correlated molecular calculations. I. The atoms boron through neon and hydrogen. J. Chem. Phys. 1989, 90, 1007-1023. 